Ann. Zootech., I968, 17 (2), 207-2II.

\title{
RELATION ENTRE LA TENEUR EN POTASSIUM, SODIUM, CALCIUM ET MAGNÉSIUM DU MUSCLE DE PORG ET CERTAINES CARACTÉRISTIQUES PHYSICO-CHIMIQUES
}

\author{
R. GOUTEFONGEA \\ avec la collaboration technique de Denise Guene et J.-P. Suquet \\ Laboratoire de Recherches sur la Viande, \\ Centre national de Recherches zootechniques, 78 -Jouy-en-Josas \\ Institut national de la Recherche agronomique
}

\section{SOMMAIRE}

La détermination des teneurs en cendres, potassium, sodium, calcium et magnésium, d'échantillons musculaires de porc, effectuée conjointement avec la mesure du $\mathrm{pH}$ et du pouvoir de rétention d'eau, montre l'existence de liaisons significatives entre la teneur en cendres et ces deux caractéristiques physico-chimiques. Par contre, les coefficients de corrélation calculés entre le $\mathrm{pH}$ et le pouvoir de rétention d'eau, d'une part, et les teneurs totales en $\mathrm{K}, \mathrm{Na}, \mathrm{Ca}, \mathrm{Mg}$, d'autre part ne sont pas significatifs.

\section{INTRODUCTION}

Le pouvoir de rétention d'eau et le $\mathrm{pH}$ du muscle sont deux des caractéristiques physico-chimiques du muscle, considérées comme des critères de la qualité de la viande. Le $\mathrm{pH}$ conditionne entre autres la qualité bactériologique des viandes et a une influence notable sur le rendement technologique; le pouvoir de rétention d'eau est à l'origine de la succulence du produit consommé et joute également un rôle important au cours des différents processus que subit la viande entre l'abattage de l'animal et la consommation : stockage, congélation et décongélation, cuisson et, en particulier, lors des transformations technologiques qui prennent place dans la fabrication des produits à base de viande.

Chez le Porc, ces deux caractéristiques musculaires sont particulièrement affectées par la myopathie exsudative et dépigmentaire. Un certain nombre d'auteurs ayant étudié ce problème ont rapporté l'existence dans les muscles exsudatifs de porc, de teneurs plus élevées en potassium (HENRY et al., r955) et en sodium (BRISKEY et al., I959) que dans les muscles normaux, résultats qui, selon les travaux de SPRAY 
et Widdowson (I950), ne peuvent d'ailleurs pas s'expliquer par d'éventuelles différences d'âge des animaux. En outre, SWIFT et BERMAN (I959) ont observé l'existence, dans le muscle de bouf, d'une relation significative entre le pouvoir de rétention d'eau et la teneur en calcium et en magnésium. Les autres résultats de la littérature concernant les relations entre teneurs en minéraux et caractéristiques physico-chimiques du muscle n'étant pas toujours concordants, nous avons réalisé cette étude dans le cas du Porc dans le but de préciser nos connaissances sur ces relations.

\section{MATÉRIEL ET MÉTHODES}

Les animaux expérimentaux, au nombre de $\mathrm{r} 3$, étaient de race Large White et provenaient de la Station de Recherches sur l'Élevage des Porcs du C. N. R. Z. Ils pesaient roo $\pm 5 \mathrm{~kg}$.

Deux heures après l'abattage, effectué selon le processus classique (électrocution, saignée, échaudage, éviscération et fente), les carcasses étaient mises en chambre froide $\grave{a}+4^{\circ} \mathrm{C}$.

Vingt-quatre heures après, le jambon gauche était séparé de la carcasse et, après dissection, un prélèvement d'environ roo $g$ était effectué sur chacun des muscles suivants :

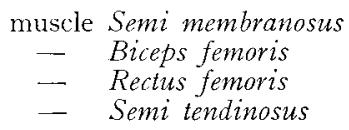

Sur ce dernier muscle, on effectuait deux prélèvements, un dans la portion claire, l'autre dans la portion foncée.

Après mesure du pH, au moyen d'un pH mètre E. I. L. $23 \mathrm{AF}_{2}$ muni d'une électrode duplex, chaque échantillon était broyé dans un hachoir équipé d'une grille à trous de $3 \mathrm{~mm}$ de diamètre et le pouvoir de rétention d'eau était déterminé par la méthode que nous avons décrite (GoUTEFonGEA, I966).

- Après détermination en double de la teneur en matière sèche par un sćjour à $105^{\circ}$ pendant 48 heures, $500 \mathrm{mg}$ de produit sec, réduit en poudre et homogénéisé, étaient minéralisés au four à $500^{\circ}$ pendant une nuit et, après pesée, repris sur bain de sable à $200^{\circ}$ par $5 \mathrm{ml}$ d'HCl pur, puis $3 \mathrm{ml}$ de $\mathrm{HNO}_{3}$ à Io $\mathrm{p}$. Ioo. Après filtration, le volume était amené à $100 \mathrm{ml}$ avec de l'eau bidistillée et la solution ainsi obtenue servait à la détermination des minéraux dosés.

Le dosage du potassium, du sodium et du calcium était effectué par spectrophotométrie de flamme à l'aide d'un appareil Eppendorf. Le dosage du magnésium, très difficile à réaliser par cette technique en raison des interférences avec le potassium et de la teneur élevée de la viande en cet ion, était effectué par la méthode de BoHuON (I962) utilisant le réactif de MANN et YOF (I956).

\section{RÉ,SULTATS - DISCUSSION}

Le tableau I, qui rassemble les moyennes des valeurs enregistrées pour les échantillons ainsi que les valeurs extrêmes, montre que l'ensemble des échantillons couvre une gamme étendue en ce qui concerne le $\mathrm{pH}$ ultime et le pouvoir de rétention d'eau.

On peut noter également une variation importante des teneurs en cendres et en $K$, $\mathrm{Na}, \mathrm{Ca}, \mathrm{Mg}$, dont les valeurs varient environ du simple au double.

Nous avons calculé les coefficients de corrélation entre les teneurs en cendres et en $\mathrm{K}, \mathrm{Na}, \mathrm{Ca}, \mathrm{Mg}$, d'une part, et les valeurs du $\mathrm{pH}$ ultime et du pouvoir de rétention d'eau, d'autre part. Les valeurs en sont rassemblées dans le tableau 2.

Les coefficients de corrélation entre la teneur en cendres et le $\mathrm{pH}$, et entre la 
teneur en cendres et le pouvoir de rétention d'eau, sont significatifs à $\mathbf{P}=0$,or. Les muscles présentant le caractère exsudatif, c'est-à-dire ayant un $\mathrm{pH}$ ultime bas et un pouvoir de rétention d'eau faible, ont une teneur en minéraux totaux plus faible.

TABLEAU I

Moyennes, écarts-types et valeurs extrêmes des données déterminées sur chaque échantillon

\begin{tabular}{|c|c|c|c|}
\hline & Valeur moyenne & Écart-type & Valeurs extrêmes \\
\hline${ }_{\mathrm{pH}}$ ultime $\ldots \ldots \ldots \ldots \ldots \ldots \ldots \ldots$ & 5,83 & 0,38 & $5,34-6,10$ \\
\hline Pouvoir de rétention d'eau en \% d'eau libre ... & 21,9 & 5,8 & $11,4-33,3$ \\
\hline Cendres (mg/g matière sèche) ............. & 46,1 & 3,7 & $31,8-62,5$ \\
\hline $\mathrm{K}$ (mg/g matière sèche)..$\ldots \ldots \ldots \ldots \ldots$ & 14,52 & 3,22 & $10,0-17,32$ \\
\hline $\mathrm{Na}$ (mg/g matière sèche) ............... & 2,26 & 0,88 & $1,21-3,49$ \\
\hline $\mathrm{Ca}$ (mg/g matière sèche)...$\ldots \ldots \ldots \ldots \ldots$ & 0,12 & 0,13 & $0,30-0,63$ \\
\hline $\mathrm{Mg}$ (mg/g matière sèche).......... & 0,91 & 0,19 & $0,74-1,75$ \\
\hline
\end{tabular}

TABLEAU 2

Coefficients de corrélation entre les teneurs en cendres et en $\mathrm{K}, \mathrm{Na}, \mathrm{Ca}, \mathrm{Mg}$ et les valeurs du pH ultime et du pouvoir de rétention d'eau

\begin{tabular}{|c|c|c|c|c|c|}
\hline & Cendres & $K$ & $\mathrm{Na}$ & $\mathrm{Ca}$ & $\mathrm{Mg}$ \\
\hline pH ultime & $+0,46^{* *}$ & $+\begin{array}{c}0,19 \\
(0,18)\end{array}$ & $+\begin{array}{c}0,18 \\
(0,20)\end{array}$ & $\begin{array}{r}-0,06 \\
(0,67)\end{array}$ & $\begin{array}{r}-0,12 \\
(0,40)\end{array}$ \\
\hline Pouvoir de rétention d'eau & $-0,44^{* *}$ & $\begin{array}{r}0,21 \\
(0,14)\end{array}$ & $\begin{array}{r}-0,12 \\
(0,40)\end{array}$ & $\begin{array}{r}0,20 \\
(0,16)\end{array}$ & $\begin{array}{r}0,10 \\
(0,48)\end{array}$ \\
\hline
\end{tabular}
dante.

** Significatif à $\mathrm{P}=0,01$.

Pour les coefficients non significatifs, nous avons mentionné entre parenthèses la probabilité correspon-

Par contre, les coefficients de corrélation entre les teneurs en les différents minéraux dosés et les deux caractéristiques physico-chimiques mesurées ne sont pas significatifs à $\mathrm{P}=0,05$. Les probabilités calculées par transformation des $\rho$ en $\mathrm{Z}$ (STEEL, et TORRIE, I960) indiquent néanmoins, pour certains de ces coefficients, une liaison qui, bien que faible, traduit une tendance à l'opposition entre les relations liant le $\mathrm{pH}$ ultime et le pouvoir de rétention d'eau aux ions monovalents, d'une part, et divalents, d'autre part. Cet antagonisme est en accord avec les résultats de HAMm (I959).

Il faut noter que, si le potassium est le minéral le plus abondant dans le muscle, le sodium, le calcium et le magnésium n'y sont présents qu'à des doses bien plus faibles et la somme des 4 éléments dosés ne représente que 55 à 60 p. Ioo des miné- 
raux totaux. Parmi les substances minérales dont nous ne nous sommes pas préoccupé ici, le phosphore est le plus important ; on sait qu'il est présent dans le muscle essentiellement sous forme de phosphates, ces derniers jouant un rôle important dans le déterminisme du pouvoir de rétention d'eau. Nous avons effectué quelques déterminations de phosphore total qui semblent montrer une teneur supétieure des muscles normaux. Ceci peut expliquer l'existence d'une liaison significative entre la teneur en cendres et le pouvoir de rétention d'eau, ainsi qu'entre la teneur en cendres et le $\mathrm{pH}$ ultime, alors que les relations entre ces deux caractéristiques physicochimiques et les teneurs en $\mathrm{K}, \mathrm{Na}, \mathrm{Ca}, \mathrm{Mg}$ ne sont pas significatives. Le rôle du phosphore et des phosphates sera précisé lors d'une étude ultérieure.

\title{
CONCLUSION
}

Bien qu'il existe des relations entre la teneur en cendres et le $\mathrm{pH}$ ultime, d'une part, et entre la teneur en cendres et le pouvoir de rétention d'eau, d'autre part, les teneurs totales du muscle de porc en potassium, sodium, calcium et magnésium ne sont pas liées significativement à ces deux caractéristiques physico-chimiques musculaires. I,es seules tendances mises en évidence semblent indiquer une certaine opposition entre alcalins et alcalins terreux en ce qui concerne leur comportement vis-à-vis du $\mathrm{pH}$ ultime et du pouvoir de rétention d'eau. Ces résultats ne signifient pas que ces minéraux ne jouent aucun rôle dans le déterminisme du $\mathrm{pH}$ et du pouvoir de rétention d'eau, mais seulement qu'au niveau de la teneur totale du tissu musculaire, aucune loi générale ne peut être dégagée.

$$
\text { Rę̧u pour publication en février } 1968 .
$$

\section{SUMMARY}

\author{
RLLATIONS BETWEEN POTASSIUM, SODIUM, CALCIUM AND MAGNESIUM CONTENTS
} OF PORK MUSCLE AND CERTAIN PHYSICO-CHEMICAL CHARACTERISTICS

Contents of ash, potassium, sodium, calcium and magnesium and also $\mathrm{pH}$ and water-retaining capacity were estimated in 65 samples of nuscle from $\mathrm{I}_{3}$ Large White pigs (table $\mathrm{I}$ ). There was a highly significant correlation between pII and water-retaining capacity on the one hand and ash content on the other. 'The respective coefficients were $r=0.46$ and $r=-0.44$. Calculated correlation coefficients between the two physico-chemical characteristics of the muscles studied and the contents of $\mathrm{K}, \mathrm{Na}, \mathrm{Ca}$ and $\mathrm{Mg}$ were not significant (table 2 ).

\section{RÉFÉRENCES BIBL,IOGRAPHIQUES}

Bohuon C., ig62. Microdosage du magnésium dans divers milieux biologiques. Clin. Chim. Acta, 7, 8 I8 I 7 .

Briskey E. J., Bray R. W., Hokkstra W. G., Phit.lips H. H., Grummer R. H., i959. The chemical and physical characteristics of various pork ham muscles classes. J. Anim. Sci., 18, I 46-1 52. 
Goutefongea R., ig66. Étude comparative de différentes méthodes de mesure du pouvoir de rétention d'eau de la viande de porc. Ann. Zootech., 15, 29I-295.

Hamm R., 1959. Biochemistry of meat hydration. Proc. 11th Res. Cont. Am. Meat Inst., Circ. 50, 1 7-23. Henry M., Billon J., Haouza G., 1955. Contribution à l'étude de l'acidose des viandes de porc, dites exsudatives. Rev. Pathol. gén. Comp., 669, 857-885.

ManN C. K., YoE J. H., 1956. Spectrophotometric determination of magnesium with sodium $\mathrm{r}-\mathrm{azo-2}$ hydroxy-3-(-2,4-dimethyl carboxanilido)-naphtalène-1'-(2-hydroxybenzène-5-sulfonate). Anal. Chem., 28, 202-206.

SpRAY C. H., WrDDowson E., 1950. The effect of growth and development on the composition of mammals. Brit. J. Nutrit., 4, 332-353.

Steel R. G. D., TORRIE J. J., I960. Principles and procedures of statistics. 434 et 452 . Mc Graw-Hill, Book Company, Inc. New York, Toronto, London.

SwifT C. E., BERMAN M. D., I959. Factors affecting the water retention in beef. I.Variations in composition and properties among eight muscles. Food Technol., 13, 365-370. 